\title{
Oscillatory Behaviors of an Epidemiological Model on Small-World Networks
}

\author{
Seung Ki BAEK* \\ Department of Physics, BK21 Physics Research Division, \\ and Institute of Basic Science, Sungkyunkwan University, Suwon 440-746
}

(Received 1 September 2006)

\begin{abstract}
We investigate the oscillatory dynamics of an epidemiological model of SIRS(susceptible-infectiverecovered-susceptible) type on small-world networks. A delay differential equation for the infected population is derived to show that three characteristic patterns, stationarity, oscillation, and synchronized extermination exist, depending on the competition between the disease's life cycle and the time for it to sweep the world. Numerical calculations support this prediction and suggest that the synchronization parameter proposed by Kuramoto can be a good measure of patterns.
\end{abstract}

PACS numbers: 87.19.Xx, 89.75.Hc, 87.23.Cc, 05.45.Xt

Keywords: Epidemic spreading, Small-world network

\section{INTRODUCTION}

Since the network became a main interest in statistical physics, the dynamics of interacting elements forming a network structure has been a hot issue [1-4]. The interacting element can be a nonlinear oscillator [5-7], an excitable neuron [8-11], a biological species [12,13], a sand pile [14-16], an epidemic disease [17-23], or even a game participant [24-26]. These approaches help one to analyze various aspects of this world, including biology, sociology, politics, and economy, as well as the physics itself [27-31].

We are interested in the small-world nature of an epidemiological model because it is widely applicable due to its simplicity. The first work of epidemiology on the small-world network [17] traced the dynamic evolution by changing the rewiring parameter $p$ and attributed the birth of oscillation to the breakdown of large clusters. In other words, the main interest was how to transit from a fixed point with fluctuations to a self-sustained oscillation. The most interesting remark is that the disease again exterminates at very high $p$ because it can find no more susceptible nodes. Later varying the contagion probability $q$ was observed to lead to three characteristic patterns: spontaneous extermination at low $q$, oscillatory synchronization at moderate $q$, and synchronized extermination at high $q$. Introducing the effect of prevention, we could reach a seemingly counter-intuitive result that prevention helps the disease survive against synchronized extermination. From these observations, we suggested that these patterns were determined by competition be-

\footnotetext{
*E-mail: garuda@skku.edu
}

tween the typical life cycle of the disease and the time taken by it to go around the world [22]. This proposition is consistent with the remark by Gade and Sinha [21] that the time scales are quite important. Roxin et al. [9] in this spirit even though they treated a neuron system.

In this brief paper, we look closer into this mechanism by changing the life cycle time of the disease and fixing other parameters. We exclude the case of a very low infection rate, resulting in spontaneous extermination, in this setup. Several consequences of the epidemiological model of concern will be presented in Section II to explain how the oscillation is developed or suppressed. After checking the numerical results in Section IV, we add some concluding remarks in the last section.

\section{ANALYSIS}

Let us consider the SIRS formalism, where the symbol $S$ indicates a susceptible state to the disease, $I$ an infected state, and $R$ a recovered state, with $N=S+I+R$ conserved. Starting with the $S$ state, a node passes to the $I$ state by infection, where it remains for a certain infection period $\tau_{I}$ and then to a recovery period $\tau_{R}$ before returning to the $S$ state. The infection occurs on a small-world network with the rewiring probability $p$ and the number of links per node $k$ with the probability of $P(S \rightarrow I)=1-(1-q)^{k_{i}}$ where $q \in[0,1]$ is the infection probability and $k_{i}$ is the number of infected neighbors. Only the nodes in the $I$ state can infect others while those in the $R$ state can not. The $R$ state actually plays the role of a barrier against the disease because it is not 
infected, either.

Before analyzing the synchronized behaviors, it is worth pointing out that oscillation is hardly possible in a regular lattice structure. The crucial reason is related to the nature of a homogeneous regular lattice in that, given a radius $r$, one can discriminate the interior from the exterior by drawing a well-defined boundary. Considering a disease with sufficiently high $q$, a closed boundary line can be drawn between the infected and the susceptible areas; thus, any infected node emits this line, propagating outward in every direction. The assumption of the SIRS model prevents a boundary from back-propagating, or penetrating another boundary line. On a finite regular lattice, henceforth, the boundary stops its propagation either at the end of the lattice or by collision with another boundary (especially under the periodic boundary condition) (Fig. 1 (a)). That the disease ceases to propagate implies that the disease cannot infect any more nodes and should die out. Even though the stochastic nature allows a nonzero probability to break the boundary line, especially in low $q$, this is negligible in a low dimension and low $q$ leads to the spontaneous extermination in most cases.

In order to avoid this triviality, we need to introduce an irregularity, destroying the boundary line between the interior and the exterior (Fig. 1 (b)). Then, the disease can return back to the susceptible regions by using the time gaps. Later, we will consider transition functions periodic in time, but they are simply impossible in a purely regular structure. One of such synchronizable disordered examples is the small-world network [17] to be considered here, and the scale-free network structure is also reported to exhibit synchronized oscillations [23].

Returning to the SIRS model, we note that once a node is infected, its evolution is completely deterministic until it returns to the $S$ state. Therefore, throughout this paper, we set $\tau_{I}=\tau_{R}=\tau$ and enjoy the equality without losing overall qualitative features:

$$
\sigma_{I}(t)=\sigma_{R}(t+\tau)
$$

where $\sigma_{Z}(t)$ is the portion of the state $Z \in\{S, I, R\}$ at time $t$. It is also evident that the following identity holds by definition:

$$
\sigma_{S}(t)+\sigma_{I}(t)+\sigma_{R}(t)=1 .
$$

Let us write down the identities to describe the evolution of the population:

$$
\begin{aligned}
& \dot{\sigma}_{S}(t)=W_{R \rightarrow S}(t)-W_{S \rightarrow I}(t), \\
& \dot{\sigma_{I}}(t)=W_{S \rightarrow I}(t)-W_{I \rightarrow R}(t), \\
& \dot{\sigma_{R}}(t)=W_{I \rightarrow R}(t)-W_{R \rightarrow S}(t),
\end{aligned}
$$

where $W_{X \rightarrow Y}$ indicates the transition rate from $X$ to $Y$. The transition from $S$ to $I$ is probabilistic and depends on the network topology, which makes it difficult to include this step. Suppose the disease appears at time $t=0$. If the recovered nodes after infection are protected

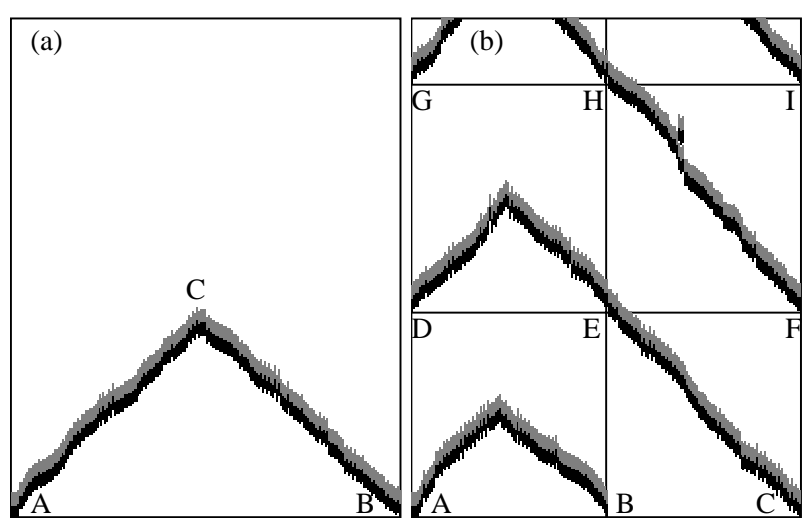

Fig. 1. Sample pictures of (a) a regular lattice and (b) a disordered lattice with $N=200, k=2, q=0.3$, and a periodic boundary conditions in common. The horizontal position represents the index of each node, where the black color means the infected, the gray the recovered, and the white the susceptible, and the time flows in the upward direction. In (a), the infection begins at the leftmost point and then spreads in both directions (A and $\mathrm{B}$ ), but the pulses collide at the point $\mathrm{C}$, followed by extermination. In (b), the disordered lattice has three shortcuts from the middle part to the leftmost point. These make the arrival times dispersed. $(\mathrm{A}, \mathrm{B}, \mathrm{C}),(\mathrm{D}, \mathrm{E}, \mathrm{F})$, or $(\mathrm{G}, \mathrm{H}, \mathrm{I})$ are directly linked together by these shortcuts, breaking the isotropy to allow the infection to occur successively.

from further infection, this step can be quantified deterministically by $\sigma_{S}(t)=\sigma_{R}(t-\tau)$. Since the protection, in reality, is far from perfect, however, the only guaranteed fact is that $\sigma_{S}(t) \leq \sigma_{R}(t-\tau)$, in general. Numerical calculations generating large amplitude oscillations indicate that the ratio between their peak heights settles down around a certain value in the long run and becomes so predictable that we may expect the infection process to repeat itself in every circulation. This limiting value depends on $p$ with a positive correlation, by which the system undergoes a phenomenon similar to the supercritical Hopf bifurcation (Fig. 2). We assume that the dynamics settles down to a repetitive pattern described by a functional form $W_{S \rightarrow I}(t)=f(t) \geq 0$. The life cycle of the disease teaches us to rewrite Eq. (3) as

$$
\begin{aligned}
& \dot{\sigma_{S}}(t)=f(t-2 \tau)-f(t), \\
& \dot{\sigma_{I}}(t)=f(t)-f(t-\tau), \\
& \dot{\sigma_{R}}(t)=f(t-\tau)-f(t-2 \tau) .
\end{aligned}
$$

Newman et al. [32] solved the spread of a disease from a single source on a small-world network by using the mean-field approximation under the continuum limit:

$$
\sigma_{I, m f}=\frac{1}{2}\left[1+s \tanh \left(\frac{2 k s}{\xi} r-\tanh ^{-1} \frac{1}{s}\right)\right]
$$

where $s=\sqrt{1+2(N k p)^{-1}}, \xi=(k p)^{-1}$, and $r$ is the path length from a randomly chosen point. The numerical estimate does not exactly coincide with this mean-field solution because the region of regular oscillations, which 
$\sigma_{\mathrm{R}}$

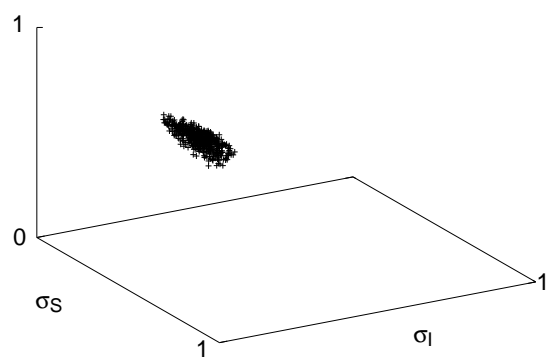

$\sigma_{\mathrm{R}}$

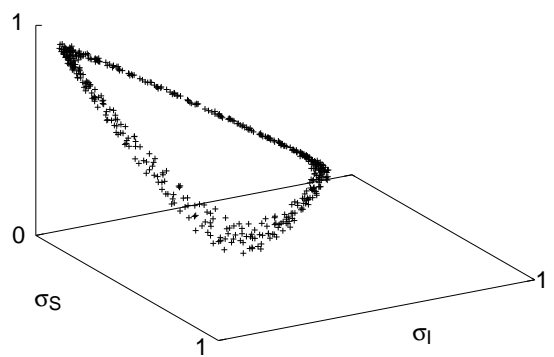

$\sigma_{\mathrm{R}}$

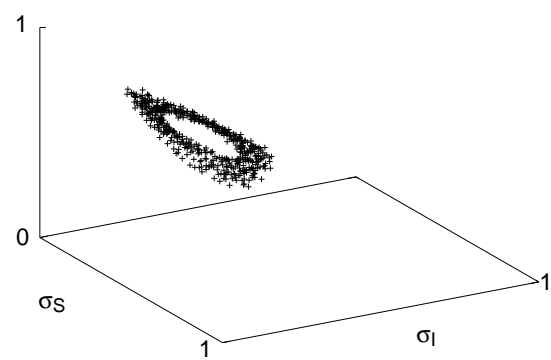

$\sigma_{\mathrm{R}}$

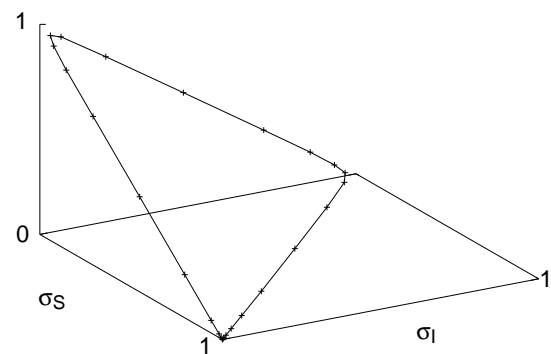

Fig. 2. Periodic trajectories at (a) $p=0.1$, (b) $p=0.2$, and (c) $p=0.5$ with $N=10^{3}, k=3$, and $\tau=7$ in common. Only 512 points are plotted after the transients are removed, and these points are confined on the plane given by Eq. (2). (d) The collapse into $\sigma_{S}=1$ can happen spontaneously or synchronously by chance. The plots from (a) to (c) are found over several realizations.

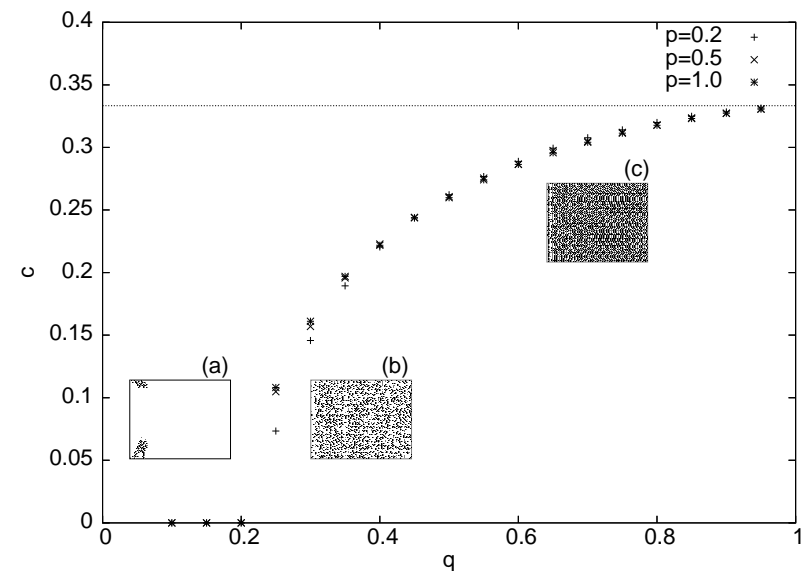

Fig. 3. Limiting proportion $c$ of the infected population as a function of $q$ when $\tau=1, N=10^{3}$, and $k=3$. (a), (b), and (c) are sample figures describing the evolution of nodes, with the time flowing in the right direction. (a) shows the simple spontaneous extermination. In the succession of spontaneous exterminations, (b), the value of $c$ varies with the randomness parameter $p$ while one can see the well-developed patterns almost independently of $p$ in (c). The line indicates $c=1 / 3$.

Kuperman and Abramson [17] reported as $p \in[0.2,0.5)$, is somewhat away from the low shortcut density limit, $\xi \ll 1$, required by the mean-field approximation. In addition, the space is not free in that the disease may often face $R$ barriers. Nevertheless, Eq. (5) captures the main qualitative shape (cf. Fig. 4), and the base function of $f(t)$ can be described by its derivative in the nearlyfree circumstances that the disease can easily find the susceptible,

$$
f_{0}(t)=\frac{k s^{2}}{\xi q} \operatorname{sech}^{2}\left(\frac{2 k s}{\xi q} t-\tanh ^{-1} \frac{1}{s}\right),
$$

where $r$ is replaced by $t / q$. At this stage, it is sufficient to focus only on its essential feature by regarding the dynamics as an effectively uniform infection, where the time taken for the disease to spread over the whole world is set to be $T_{s}$. Then, we classify three different cases from a simple comparison between time scales.

The first case is what we have called synchronized extermination when $T_{s}<\tau$. $\sigma_{I}$ reaches one at $t=T_{s}$, decreases from $t=\tau$, and finally exterminates at $t=\tau+T_{s}$. The $S$ states reappear at $t=2 \tau>\tau+T_{s}$ when no disease exists. If the network topology taken into account, $\sigma_{I}$ is not necessarily zero at $t=2 \tau$, but the point is that $S$ and $I$ do not meet each other at all.

The second case is given by $\tau<T_{s}<2 \tau$. $\sigma_{I}$ reaches almost $\tau / T_{s}$ at the first peak, and the $S$ nodes reappear before all $I$ 's die out, whereby a self-sustained oscillation begins to be possible. Since it may take some time to find susceptible nodes, the period $T$ can be a little larger than $2 \tau$, but not larger than $3 \tau$. Eq. (4) says that the Fourier 

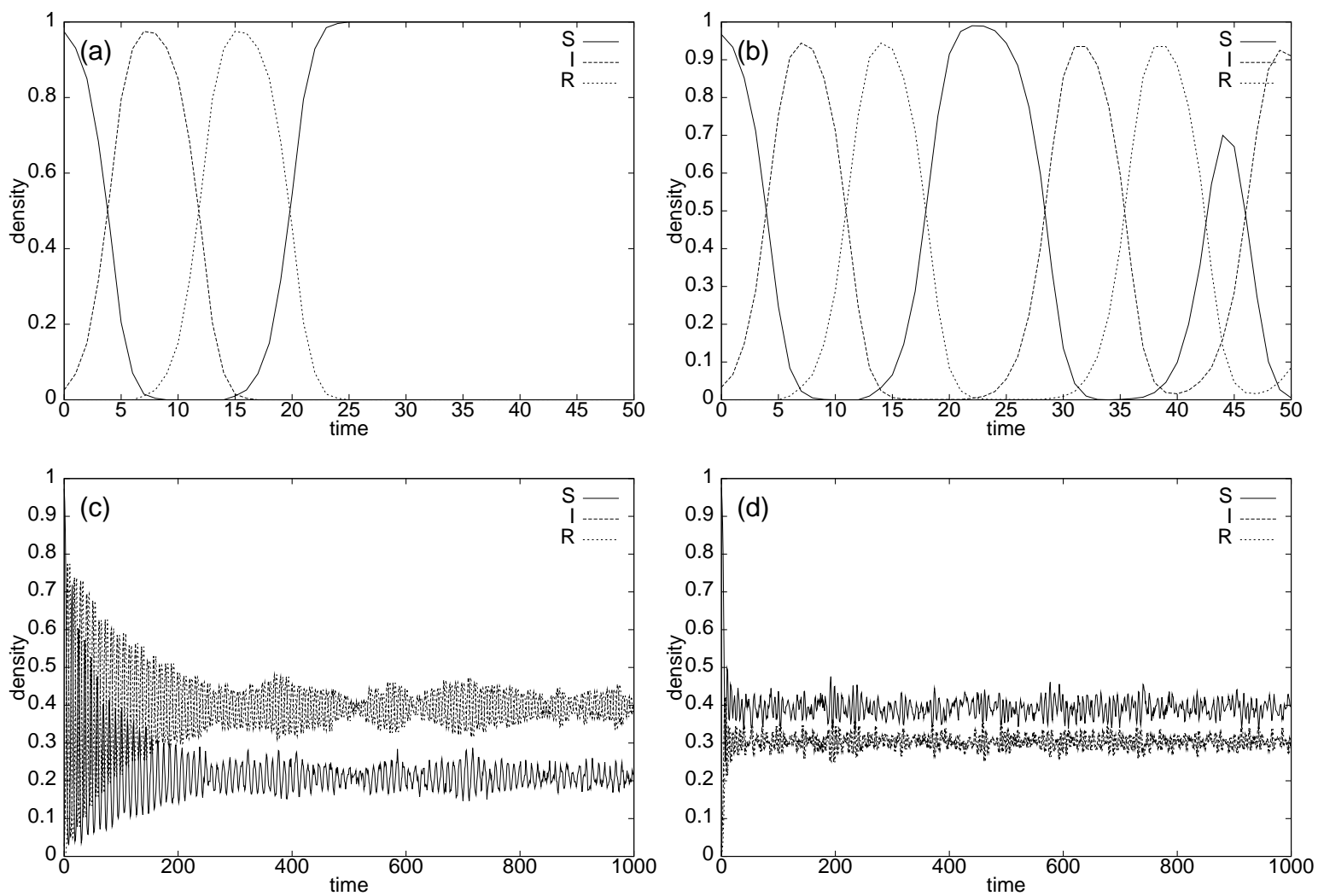

Fig. 4. Varying $\tau$ with $N=10^{3}, k=3, p=0.5$, and $q=0.3$. (a) Synchronized extermination due to the fact that the disease has too slow a life cycle $(\tau=8)$ compared with the sweeping time $T_{s}$. The peak almost booms up to one, but it fails to enter the oscillation. (b) The life cycle is set to be slightly faster $(\tau=7)$. The disease barely avoids extermination during the interval $t \in[15,22]$ and starts to oscillate in a persistent way. (c) $\tau=4$. As the whole life cycle becomes comparable with the sweeping time, the oscillatory motion is more suppressed than before. (d) With $\tau=2$, the motion is just stationary with noisy fluctuations.

components are related by

$$
\tilde{f}(\omega)=\frac{1}{1-e^{-i \omega \tau}} \tilde{\dot{\sigma}}_{I}(\omega)
$$

therefore, $f(t)$ is of period $T$, i.e.,

$$
f(t)=\sum_{n} f_{0}(t-n T)
$$

in this case. Such periodicity directly indicates that

$$
\begin{aligned}
\int_{t}^{t+T}\left\{f\left(t^{\prime}\right)-f\left(t^{\prime}-\tau\right)\right\} d t^{\prime} \\
\quad=\int_{t}^{t+T} \dot{\sigma}_{I}\left(t^{\prime}\right) d t^{\prime}=\sigma_{I}(t+T)-\sigma_{I}(t)=0
\end{aligned}
$$

for any given $t$ and $\tau$. Additionally, as $\tau$ decreases, the peak height also decreases as one can check in the meanfield approximation that $\max \{\tanh (t)-\tanh (t-\tau)\}=$ $2 \tanh (\tau / 2) \rightarrow 0$ with $\tau \rightarrow 0$.

Lastly, the remaining case is $T_{s}>2 \tau$. Low $\tau$ affects the oscillation in two ways: Firstly, even before the infected population decreases, the recovery period ends, and new susceptible nodes are supplied. In other words, the generations are overlapped, and some transition is expected. In addition, an infected node has little time to infect others. This second effect can be compensated for by increasing $q$ and we see a high frequency oscillation, which indicates generation overlapping (Fig. 3(c)). Unless $q$ is increased, the dynamics is described by a succession of spontaneous exterminations (Fig. 3(b)). Without a sufficient small-world effect, the disease cannot have enough descendants and just decays out (Fig. 3(a)). Denoting the limiting number of the infected population as $c$, it depends on $p$ in phase (b) and has an upper limit of about $1 / 3$. We can say that the average probability of infection, $p_{a v g}$, is an increasing function of $q$. In the stationary state, $\sigma_{R}$ is equal to $\sigma_{I}=c$ from Eq. (1), and $\sigma_{S}=1-2 c$. From the small-world property, almost every susceptible node is expected to have at least one infected neighbor and can be infected with a probability of around $p_{\text {avg }}$. After $\tau=1$, these new infected nodes will maintain the value of $c$. In short, the following relation is established:

$$
\begin{aligned}
& p_{\text {avg }}(1-2 c)=c, \\
& c=\frac{p_{\text {avg }}}{2 p_{\text {avg }}+1} \leq \frac{1}{3} .
\end{aligned}
$$



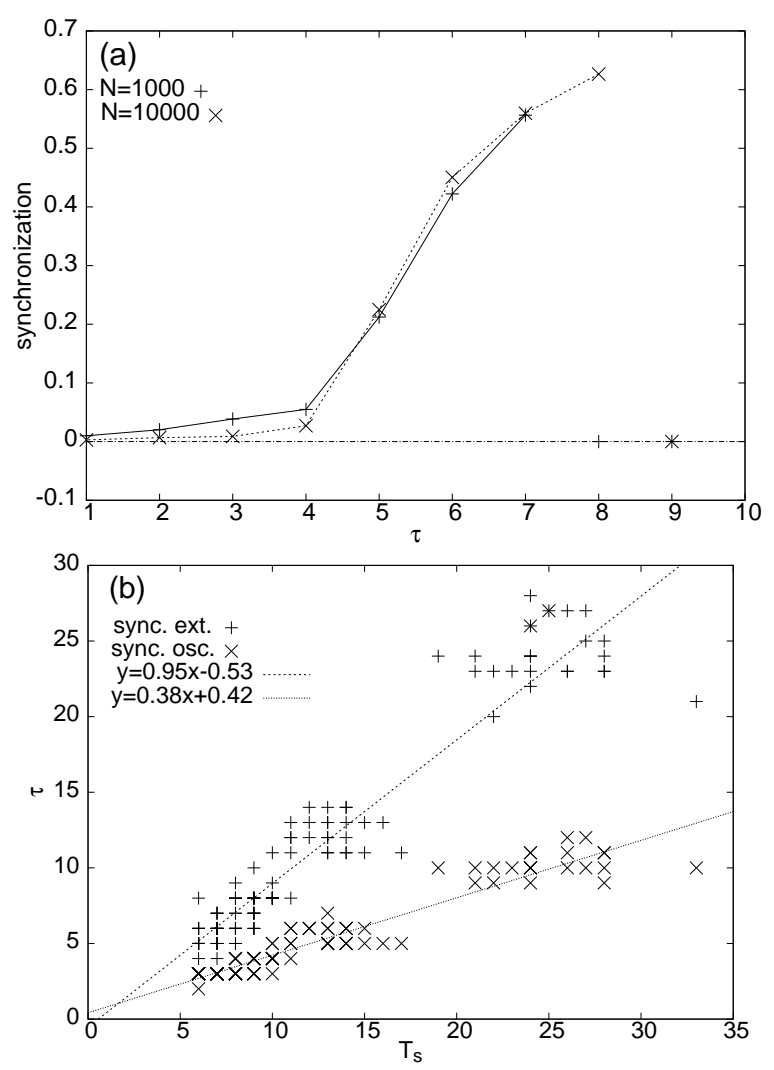

Fig. 5. (a) Synchronization parameter $\gamma$ as a function of $\tau$, averaged over 500 time steps at $k=3, p=0.5$, and $q=0.3$. When oscillations develop, $\gamma$ shows significant increases ( $\tau=4$ in both cases). $\gamma$ is identically zero at high $\tau$ values due to extermination $\left(\tau=8\right.$ with $N=10^{3}$ and $\tau=9$ with $N=10^{4}$ ). (b) Relationships between $T_{s}$ and the transition points. The horizontal axis means $T_{s}$, and the vertical positions represent the values of $\tau$ where the synchronized exterminations occur $(+)$ and where the synchronized oscillations are established $(\times)$. Here, $T_{s}$ is regarded as the time taken for over $90 \%$ of the whole population to have ever been infected, and the critical value of $\gamma$ to detect the synchronized oscillations is set to be 0.1 . The transition points to the synchronized extermination are identified with $\tau$ 's where where nonzero $\gamma$ first appears from 30 realizations, as $\tau$ decreases from above. $p$ and $q$ vary within $[0.3,1.0]$ and $[0.1,0.5]$, respectively.

where the equality holds when $p_{\text {avg }}=1$. We carry out a sort of mean-field calculation in analogy with the chemical reactions in Appendix as a qualitative example, though it does not exactly coincide with the above results.

\section{NUMERICAL RESULTS}

Some numerical results are depicted in Fig. 4 for $N=10^{3}, k=3, p=0.5$, and $q=0.3$ in common. Fig. 4(a) corresponds to the first case of $T_{s}<\tau$, classified above as synchronized extermination. The infected population reaches nearly $100 \%$ at the first peak, showing an extreme synchronization, but the circulation is maintained only one time. Note that this direct simulation shows $T_{s} \simeq 8$. A little smaller $\tau$ enables large amplitude oscillations to occur repeatedly (Fig. 4(b)), as many authors have already reported. The oscillation does not retain its shape in much smaller $\tau$ (Fig. 4(c)) and transits gradually to a noisy fluctuation in the end (Fig. 4(d)). Our analysis in the previous section suggests that the oscillation cannot be sustained in the mean-field limit where every node interacts with the others with different states on average and that the situation actually could correspond to the generation overlapping.

To quantify the characteristic behaviors, we check the transition of the synchronization parameter [33]

$$
\gamma=\left|\frac{1}{N} \Sigma_{j=1}^{N} e^{i \phi_{j}(t)}\right|
$$

where $\phi_{j}$ is the phase of the disease's life cycle at the node $j$. The $S$ state corresponds to $\phi_{j}=0$ and is excluded from the summation [17]. Fig. 5(a) shows the typical results, and we can see three kinds of the characteristic behaviors, stationarity, oscillation, and synchronized extermination. If we regard those points with zero synchronizations as signs of exceeding $T_{s}, T_{s}$ is estimated roughly as 8 in the case of $N=10^{3}$ and as 9 in the case of $N=10^{4}$, consistent with the fact that the characteristic path length scales as $O(\log N)$ [34].

Synchronized oscillations are expected to exist in $T_{s} / 2<\tau<T_{s}$, according to the previous section. Although the probabilistic nature makes it difficult to analyze, this relation seems to hold over a variety of parameter sets (Fig. 5(b)).

The least-squares fitting shows that the proportionalities of critical $\tau$ with respect to $T_{s}$ are given by 0.38 and 0.95 , which are not very far from the predicted values $1 / 2$ and 1 , even though the estimate can be affected by the criteria we take to determine the transition points and $T_{s}$.

\section{CONCLUSION}

We examined the oscillation of the SIRS model on a small-world network from the viewpoint of the competition between two time scales: the life cycle of the disease and the sweeping time. Some sketches of the mathematical treatment of this system were given in terms of the delay differential equation, by which we could observe three characteristic patterns, stationarity, oscillation, and synchronized extermination, once the disease had enough ability of infection.

The SIRS formalism requires only synchronizable elements having some inactive period after infection; thereby, we can say that it is quite a general model. Similar patterns appear in many fields, including ecology and economics, and understanding this dynamic is 
an important task. The solution space is searched, not exhaustively here but only roughly, to provide an intuitive understanding, and more rigorous work remains to be done.

\section{APPENDIX}

When the states are desynchronized, we may assume that this randomly mixed up situation can be approximated by a sort of the chemical-reaction type interaction $S+I \longrightarrow I+I$. The transition function is given by

$$
f(t) \propto N q \sigma_{S}(t) \sigma_{I}(t),
$$

where the proportionality coefficient is related to the reaction frequency within a given time unit, and we normalize it to unity in accordance with the discrete version. Eq. (4) becomes

$$
\begin{aligned}
& \dot{\sigma}_{I}(t) \simeq N q\left\{\sigma_{S}(t) \sigma_{I}(t)-\sigma_{S}(t-\tau) \sigma_{I}(t-\tau)\right\} \\
& \quad=N q\left[\left\{1-\sigma_{I}(t)-\sigma_{I}(t-\tau)\right\} \sigma_{I}(t)\right. \\
& \left.\quad-\left\{1-\sigma_{I}(t-\tau)-\sigma_{I}(t-2 \tau)\right\} \sigma_{I}(t-\tau)\right] .
\end{aligned}
$$

It is easy to see that Eq. (A.1) has a stationary solution. We approximate Eq. (A.1) by a set of ordinary differential equations:

$$
\begin{aligned}
& \dot{x}=N q[(1-x-y) x-(1-y-z) y], \\
& \dot{y}=\tau^{-1}(x-y), \\
& \dot{z}=\tau^{-1}(y-z) .
\end{aligned}
$$

$x(t)$ represents the variable $\sigma_{I}(t)$ of our main concern, and $y(t)$ is introduced to trace $x(t)$ with a characteristic time scale $\tau$ mimicking $x(t-\tau)$ as $z(t)$ does $y(t-\tau)$. The fixed point is $(x, y, z)=(c, c, c)$ for a certain constant $c \in\left(0, \frac{1}{2}\right]$ where the upper limit is required by Eq. (2).

The eigenvalues are found by solving the following characteristic polynomial equation:

$$
\begin{aligned}
& \left(1-3 c-\frac{\lambda}{N q}\right)\left(\tau^{-1}+\lambda\right)^{2} \\
& \quad+(2 c-1) \tau^{-1}\left(\tau^{-1}+\lambda\right)+c \tau^{-2}=0 .
\end{aligned}
$$

One can readily solve this equation for $\lambda$ to find the stability criterion of the fixed point. The set of equations approaches the original delay differential equation if the dimensionality increases by introducing more variables $\dot{y}_{\alpha}=m \tau^{-1}\left(y_{\alpha-1}-y_{\alpha}\right), \alpha=1, \cdots, 2 m$ with $y_{0}=x$. In the limit $m \rightarrow \infty$, these equations, as a whole, correspond to a partial differential equation $(\partial y / \partial \alpha)+\tau(\partial y / \partial t)=0$, which admits a propagating solution of the form $g(t / \tau-\alpha)$. The equation of $x$ then also changes into

$$
\dot{x}=N q\left[\left(1-x-y_{m}\right) x-\left(1-y_{m}-y_{2 m}\right) y_{m}\right],
$$

and the characteristic equation is obtained as

$$
\begin{aligned}
& \left(1-3 c-\frac{\lambda}{N q}\right)\left(m \tau^{-1}+\lambda\right)^{2 m}+(2 c-1)\left(m \tau^{-1}\right)^{m} \\
& \quad \times\left(m \tau^{-1}+\lambda\right)^{m}+c\left(m \tau^{-1}\right)^{2 m}=0 .
\end{aligned}
$$

As $m \rightarrow \infty$, we get a transcendental equation

$$
(2 c-1) e^{-\lambda \tau}+c e^{-2 \lambda \tau}=\frac{\lambda}{N q}+3 c-1 .
$$

In the limit of infinite $N$, the first term on the right-hand side(RHS) vanishes, and we get

$$
(2 c-1) e^{-\lambda \tau}+c e^{-2 \lambda \tau}=3 c-1 .
$$

Let $\lambda$ be a general complex number $a+b i$, where $a$ and $b$ are both real. Then, Eq. (A.3) yields a set of equations,

$$
(2 c-1) e^{-a \tau} \cos b \tau+c e^{-2 a \tau} \cos 2 b \tau=3 c-1,
$$

and

$$
(2 c-1) e^{-a \tau} \sin b \tau+c e^{-2 a \tau} \sin 2 b \tau=0,
$$

where the first equation is for the real part, and the second one for the imaginary part. Eq. (A.5) gives us two choices: $\sin b \tau=0$ or not. If we consider the nonzero case first, Eq. (A.5) reduces to

$$
e^{-a \tau} \cos b \tau=\frac{1}{2 c}-1 \text {. }
$$

Substituting this in Eq. (A.4), we obtain

$$
\cos ^{2} b \tau=\frac{(1-2 c)^{2}}{-12 c^{2}+4 c} \text {. }
$$

A square of cosine should lie in $[0,1]$, but the RHS of Eq. (A.6) cannot satisfy this requirement unless $c=1 / 4$. Since then $\cos ^{2} b \tau=1$, it contradicts the assumption that $\sin b \tau \neq 0$. On the other hand, if $\sin b \tau=0$, which is equivalent to $\cos b \tau= \pm 1$, Eq. (A.4) yields

$$
a=-\frac{1}{\tau} \log \left|\frac{1}{c}-3\right| .
$$

Therefore, a positive $a$ can be found for $\frac{1}{4}<c<\frac{1}{2}$.

We did not determine the value of $c$ yet. The answer comes from the balance between the input and the output. In the stationary state, the age structure of the infected population is supposed to be uniform, and the portion to enter the $R$ state is roughly $1 / \tau$. Henceforth,

$$
\begin{aligned}
& \frac{1}{\tau} c=N q(1-2 c) c, \\
& c=\frac{1}{2}\left(1-\frac{1}{N \tau q}\right) .
\end{aligned}
$$

As $N \rightarrow \infty, c$ approaches $1 / 2$. This is natural because any susceptible node will be soon infected through the mean-field-like reaction. From the above calculation, we know that $c=1 / 2$ does not give an eigenvalue with a positive real part. The value, however, is not the same as the actual simulation result.

\section{ACKNOWLEDGMENTS}

This work was supported by the Korea Research Foundation Grant funded by the Korean Government(MOEHRD) (KRF-2005-005-J11903). 


\section{REFERENCES}

[1] D. H. Watts and S. H. Strogatz, Nature (London) 393, 440 (1998).

[2] R. Albert, H. Jeong and A.-L. Barabási, Nature (London) 401, 130 (1999).

[3] H. Jeong, B. Tombor, R. Albert, Z. N. Oltvai and A.-L. Barabási, Nature (London) 407, 651 (2000).

[4] R. Albert and A.-L. Barabási, Rev. Mod. Phys. 74, 47 (2002).

[5] L. F. Lago-Fernández, R. Huerta, F. Corbacho and J. A. Signenza, Phys. Rev. Lett. 84, 2758 (2000).

[6] H. Hong, M. Y. Choi and B. J. Kim, Phys. Rev. E 65, 026139 (2002).

[7] H. Hong, B. J. Kim, M. Y. Choi and H. Park, Phys. Rev. E 69, 067105 (2004).

[8] O. Kwon and H.-T. Moon, Phys. Lett. A 298, 319 (2002).

[9] A. Roxin, H. Riecke and S. A. Solla, Phys. Rev. Lett. 92, 198101 (2004).

[10] H. Hasegawa, Phys. Rev. E 70, 066107 (2004).

[11] O. Kwon, H.-H. Jo and H.-T. Moon, Phys. Rev. E 72, 066121 (2005).

[12] S. Lee and Y. Kim, Phys. Rev. E 71, 057102 (2005).

[13] S. Lee and Y. Kim, J. Korean Phys. Soc. 48, 249 (2006).

[14] D.-S. Lee, K.-I. Goh, B. Kahng and D. Kim, Physica A 338, 84 (2001).

[15] R. Karmakar and S. S. Manna, J. Phys. A: Math. Gen. 38, L87 (2005).

[16] K. Rho, S. R. Hong and B. Kahng, J. Korean Phys. Soc. 47, L558 (2005).

[17] M. Kuperman and G. Abramson, Phys. Rev. Lett. 86, 2909 (2001).

[18] Y. Moreno, R. Pastor-Satorras and A. Vespignani, Eur.
Phys. J. B 26, 521 (2002).

[19] N. Madar, T. Kalisky, R. Cohen, D. ben-Avraham and S. Havlin, Eur. Phys. J. B 38, 269 (2004).

[20] M. Barthelemy, A. Barrat, R. Pastor-Satorras and A. Vespignani, J. Theor. Biol. 235, 275 (2005).

[21] P. M. Gade and S. Sinha, Phys. Rev. E 72, 052903 (2005).

[22] H.-H. Jo, S. K. Baek and H.-T. Moon, cond-mat/ 0310372; Physica A 361, 534 (2006).

[23] G. Yan, Z.-Q. Fu, J. Ren and W.-X. Wang, physics/ 0602137 (2006).

[24] M. Anghel, Z. Toroczkai, K. E. Bassler and G. Korniss, Phys. Rev. Lett. 92, 058701 (2004).

[25] T. S. Lo, K. P. Chan, P. M. Hui and N. F. Johnson, Phys. Rev. E 71, 050101 (2005).

[26] S. H. Lee and H. Jeong, J. Korean Phys. Soc. 48, 186 (2006).

[27] P.-J. Kim and H. Jeong, J. Korean Phys. Soc. 44, 621 (2004).

[28] W. S. Jung, O. Kwon, J.-S. Yang and H.-T. Moon, J. Korean Phys. Soc. 48, 135 (2006).

[29] K. H. Chang, K. Kim, H. Oshima and S.-M. Yoon, J. Korean Phys. Soc. 48, 143 (2006).

[30] S.-W. Son and H. Jeong, J. Korean Phys. Soc. 48, 208 (2006).

[31] H.-J. Youn, H. Jeong, F. Roth, M. Silver, M.-H. Clutier and P. Ittzes, J. Korean Phys. Soc. 48, 217 (2006).

[32] M. E. J. Newman, C. Moore and D. J. Watts, Phys. Rev. Lett. 84, 3201 (2000).

[33] S. H. Strogatz, Physica D 143, 1 (2000).

[34] B. J. Kim, H. Hong and M. Y. Choi, Phys. Rev. E 68, 014304 (2003). 